\title{
Temperature-water and competitive interactions in vitro of two toxic black molds, Fusarium oxysporum and Rhizopus stolonifer isolated from a shower setting
}

\section{Yoder JA, Klever LA and Dobrotka CJ}

Department of Biology, Wittenberg University, Springfield, OH, 45504, USA

Yoder JA, Klever LA, Dobrotka CJ 2018 - Temperature-water and competitive interactions in vitro of two toxic black molds, Fusarium oxysporum and Rhizopus stolonifer isolated from a shower setting. Studies in Fungi 3(1), 248-255, Doi 10.5943/sif/3/1/25

\begin{abstract}
We report differences in secondary resource capture and upper lethal temperature as survival attributes for Fusarium oxysporum (FOSC) and Rhizopus stolonifer, two medically important fungi that were co-isolated from a table shower caddy. Isolates of these two shower-associated fungi have been deposited at the University of Alberta Microfungus Collection and Herbarium, Centre for Global Microfungal Biodiversity (UAMH, Toronto, CAN) as $R$. stolonifer UAMH 11965 and $F$. oxysporum (FOSC) UAMH 11966; this paper provides growth characteristics for these two strains that can be used for further studies on black molds in bathrooms given the relevance to public health. Both $R$. stolonifer UAMH 11965 and $F$. oxysporum (FOSC) UAMH 11966 require contact with a wet surface substrate with water activity $\geq 0.95 \mathrm{a}_{\mathrm{w}}$ for growth and sporulation. In contrast to $F$. oxysporum, $R$. stolonifer has a 4-6x, faster radial growth rate, superior colonizing ability, and capacity for overgrowth (exploitative competition). This $F$. oxysporum (FOSC) strain, however, is thermotolerant, as demonstrated by broader thermal growth range and higher optimum temperature, which puts fewer limitations on its growth compared to $R$. stolonifer. This is a point of public awareness for mold-sensitive and immunocompromised people that wet, wood substrates in a shower can permit colonization and competitive interactions that can concentrate $R$. stolonifer and $F$. oxysporum spores.
\end{abstract}

Key words - Bathroom - fungal ecology - public health - radial growth - resource capture

\section{Introduction}

High relative humidity, moisture, warm temperature, soap and shampoo surfactants create an ideal habitat for a variety of pathogenic and allergenic molds to people (Hamada \& Abe 2009, 2010a,b, Short et al. 2011). Acremonium, Alternaria, Aureobasidium, Cladosporium, Cyphellophora, Exophiala, Fusarium, Paecilomyces, Phoma, Ramichloridium, Rhodotorula, and Scolecobasidium (ascomycetes and basidiomycetes) are some of the medically important molds that have been recovered from bathroom settings (Hamada \& Abe 2009, 2010a,b). Of these, the most noteworthy and frequent among the bathroom molds is Fusarium, a genus that consists of numerous potentially harmful species (Short et al. 2011, Levetin et al. 2016, Egbuta et al. 2017). There are few reports of bathroom contamination with zygomycetes. However, Garner \& Machin (2008) reported Rhizopus pusillus with a high frequency of isolation from a patient's shower in connection with water damage, air contamination, and in association with wooden substrates. 
Under adequate moisture and temperature conditions, any of these fungal bathroom contaminants are capable of producing copious amounts of spores, conidia and sporangiospores (Amari et al. 2011). Children, the elderly, mold-sensitive, and immunocompromised people, in particular, are at an elevated risk of infection via spores. The spores gain access to the inside of the body by handling, inhalation, introduction into broken skin, or by way of wounds (Egbuta et al. 2017). Although seldom reported in clinical literature, dual infections in skin and respiratory system can take place, including harmful Rhizopus-Fusarium combinations (de Almeida Júnior et al. 2016). A Rhizopus-Fusarium combination also occurs in decomposing potatoes (Ibrahim et al. 2014).

Here, we report a Rhizopus-Fusarium growth that was isolated from a wood table caddy that was being used in a bathroom shower. The two fungi were identified as Rhizopus stolonifer UAMH 11965 and Fusarium oxysporum species complex (FOSC) UAMH 11966. We have chosen to keep the company of the wood shower table caddy confidential. This specific source information is not pertinent to this investigation, only that a wood table shower caddy can be a substrate for Rhizopus and Fusarium fungal growth in a shower. Because this $R$. stolonifer strain, and similarly this $F$. oxysporum strain, were isolated from a shower setting make these strains of particular public health value in relation to bathroom hygiene.

This study compares the growth characteristics of $R$. stolonifer UAMH 11965 and $F$. oxysporum (FOSC) UAMH 11966, with these goals: 1. to determine how each fungus grows in the presence of each other (i.e., combative strategy); and 2. to define the factors that are limiting to fungus growth and sporulation (water availability and temperature requirements). We are testing the hypothesis that these $F$. oxysporum and $R$. stolonifer strains may differ in their water and temperature requirements.

\section{Materials \& Methods}

\section{Fungus isolation and identification}

Random block design was used to select three different sites from a patch of mold that had developed on the undersurface of the wood table shower caddy. Each site was swabbed with three, individual cotton tipped applicators (Puritan Medical Products, Guilford, ME). The sample was streaked for isolation onto Potato Dextrose Agar (PDA) in a 100 x 15 mm Petri dish (Fisher Scientific, St. Louis, MO). A total of nine Petri dishes were inoculated from the test mycelium. Incubation was in darkness, $25 \pm 0.5^{\circ} \mathrm{C}$ (Percival incubator, Perry, IA). Isolations were made by subculturing hyphal tips from the advancing edge of isolated colonies (40-45x light microscopy). Each hyphal tip was considered an isolate. Isolates were identified at the University of Alberta Microfungus Collection and Herbarium, Centre for Global Microfungal Biodiversity (UAMH, Toronto, CAN).

All experiments were conducted on PDA, which is the medium of choice for maintaining stable spore size/shape, hyphal branching pattern, hyphae diameter, and colony pigmentation in Rhizopus (Hernádez-Lauzardo et al. 2005) and Fusarium (Rafique et al. 2015). Design of temperature-water activity, growth, and competition experiments follows standard methods (Lockwood 1992, Wicklow 1992, Baldrian \& Gabriel 2002).

\section{Determination of optimum temperature and water activity for growth}

Computer-programmable incubators $\left(\mathrm{SD}< \pm 0.5^{\circ} \mathrm{C}\right.$; Percival) were used to control temperature. Glycerol (> 99.5\% pure; Sigma Chemicals, St. Louis, MO)-supplemented PDA was used to adjust the water activity $\left(a_{w}\right)$ of the agar media (Rousseau \& Donèche 2001). Each water activity was measured using a hygrometer ( $\mathrm{SD} \pm 0.005 \% \mathrm{a}_{\mathrm{w}}$; Thomas Scientific, Philadelphia, PA). Based on interpretations according to Christian (1980), the water activity of pure water $=1.00 \mathrm{a}_{\mathrm{w}}$, the moisture content of a surface with a water activity $\geq 0.90 \mathrm{a}_{\mathrm{w}}$ is "wet"; and the moisture content of a surface with a water activity $0.65-0.85 \mathrm{a}_{\mathrm{w}}$ is "damp".

All experiments were conducted on solidified media in $100 \times 15 \mathrm{~mm}$ Petri dishes (Fisher) that had been scored into quadrants by drawing two lines that crossed each other on the bottom of the dish. Two-week old cultures of each test fungus were used, using a $0.5 \mathrm{~cm}^{2}$ agar plug, made with the 
end of a sterile pipette (Fisher) from the edge of the mycelium. The plug was placed on top solidified PDA at the center of the dish, overtop the intersection of the two lines that had been drawn on the bottom. Dishes were incubated and examined daily by 40-45x light microscopy. Five measurements were taken on each line $(n=4)$ as the mycelium spread over the agar surface; $n=20$ growth measurements/dish, set up in triplicate; thus, each growth rate is the mean $( \pm \mathrm{SE})$ of 60 individual measurements. Plain agar plugs containing no fungus served as controls.

Radial growth rate $\left(\mathrm{K}_{\mathrm{r}}\right)$ was calculated: $\mathrm{K}_{\mathrm{r}}=\left(\mathrm{R}_{1}-\mathrm{R}_{0}\right) /\left(\mathrm{t}_{1}-\mathrm{t}_{0}\right)$, where $\mathrm{R}_{1}$ and $\mathrm{R}_{0}$ are colony radii at the beginning of the linear $\left(\mathrm{t}_{0}\right)$ and stationary $\left(\mathrm{t}_{1}\right)$ phases of growth. Experimental growth period was ten days or until five measurements had been taken. Radial growth rates were compared using an analysis of covariance (ANCOVA; $p<0.05$ ) and response surface methodology for examination of optimization effects of combined temperature and water activity (SPSS 14.0 Microsoft Excel and Minitab, Chicago, IL).

\section{Determination of competitive ability by primary resource capture}

Two $0.5 \mathrm{~cm}^{2}$ agar plugs of fungus (from two-week old culture) were placed opposite each other in a $100 \times 15 \mathrm{~mm}$ Petri dish on solidified PDA. Each plug was placed $5 \mathrm{~mm}$ from the edge of the dish. Four lines for measuring the spread of the mycelium over the agar surface were drawn on the bottom of the dish such that the lines (separated by $36^{\circ}$ from each other) radiated outward from the center of the plug. The two fungi were growing at the same time, opposite each other, in the dish. Growth measurements were stopped before the fungal hyphae made contact with each other, as determined by $40 / 45 \mathrm{x}$ light microscopy. Dishes were incubated at $25^{\circ} \mathrm{C}$. The radial growth rate $\left(\mathrm{K}_{\mathrm{r}}\right)$ was calculated as described above for both fungi in the pairing as the mycelium of each approached the other.

The experiment was conducted in triplicate; i.e., each radial growth rate is the mean $( \pm \mathrm{SE})$ of 20 measurements (five per growth line), replicated three times, to total $n=60$ individual measurements. Data were compared with ANCOVA. In all cases, microscopic examination of the mycelium margin, and hyphal, spore, and colony characteristics were used to track and distinguish each fungus.

\section{Determination of competitive ability by secondary resource capture}

A $0.5 \mathrm{~cm}^{2}$ agar plug of fungus (inoculum), taken from a two-week old culture, was placed on top, at the center, of an established mycelium that had been growing in a Petri dish $(100 \times 15 \mathrm{~mm})$ on solidified PDA for one week. The Petri dish had been scored with two lines such that the dish was divided into quadrants. Measurements were taken, as described above, along the lines for the fungus inoculum as it spread over the existing mycelium. Measurements were used to calculate the radial growth rate $\left(\mathrm{K}_{\mathrm{r}}\right)$ of the inoculum as described above. Fungi were differentiated based on spore and colony characteristics, mycelium margin, and pure culture comparison by 40/45x microscopy. No fungal growth, $\mathrm{K}_{\mathrm{r}}=0.00 \mathrm{~mm} /$ day, was confirmed by 40/45x microscopy, as an absence of visible hyphae and subsequent spores. Each experiment was replicated three times, for a total $n=60$ individual measurements for the growth rate of each fungus. ANCOVA $(p<0.05)$ was used to compare the radial growth rates (the mean $\pm \mathrm{SE} ; p<0.05$ ).

\section{Determination of competitive ability by differential competition}

Twenty $0.5 \mathrm{~cm}^{2}$ plugs of fungus were placed $1 \mathrm{~cm}$ apart from each other in a $100 \mathrm{x} 15 \mathrm{~mm}$ Petri dish on solidified PDA. The twenty plugs of fungus represented different proportions of the two fungus strains in each Petri dish. One strain was designated as fungus $\mathrm{A}$ and the other strain as fungus B. The plugs of different fungi were placed into the dish by random block design. The perimeter of the mycelium around each plug was measured daily with a digital planimeter (Professional Equipment Inc., Hauppauge, NY). Of 20 plugs of fungus in a Petri dish, test proportions were: $1.0=$ 20 plugs fungus $\mathrm{A} ; 0.8=16$ plugs fungus $\mathrm{A}$ and 4 plugs fungus $\mathrm{B} ; 0.6=12$ plugs fungus $\mathrm{A}$ and 8 plugs fungus $\mathrm{B} ; 0.4=8$ plugs fungus $\mathrm{A}$ and 12 plugs fungus $\mathrm{B} ; 0.2=4$ plugs fungus $\mathrm{A}$ and 16 plugs fungus $\mathrm{B}$; and $0.0=20$ plugs fungus $\mathrm{B}$. 
A 0.5 proportion (10 plugs fungus $\mathrm{A}$ and 10 plugs fungus $\mathrm{B}$ ) was used to calculate the relative crowding coefficient, $\mathrm{RCC}=[$ (area occupied by fungus $\mathrm{A}$ at $0.5: 0.5) /($ area occupied by fungus $\mathrm{B}$ at $0.5: 0.5)] /[($ area occupied by fungus $\mathrm{A}$ at 1:0)/(area occupied by fungus $\mathrm{B}$ at 1:0)]. If fungus $\mathrm{A}$ has an advantage over fungus $\mathrm{B}$, then the RCC $>1$. Conversely, an RCC $<1$ means that fungus $\mathrm{B}$ is a better competitor than fungus A. Results were compared using ANCOVA $(p<0.05)$, based on three replicates of each proportion of fungal species tested.

\section{Results}

\section{Fungus isolation and identification}

At the sampling site where mold was growing, 14 colonies were found at the first area swabbed, six at the second area swabbed, and 19 from the third area swabbed $(n=3$ replicates at each area swabbed; $n=9$ ) for a total of 39 isolations. The percentage breakdown of isolates was $62 \pm 1.1 \%$ Rhizopus, $33 \pm 0.4 \%$ Fusarium, and $5 \pm 0.5 \%$ Penicillium (the mean $\pm \mathrm{SE} ; \mathrm{P}<0.05$ ). One isolate was identified as Mycelia sterilia. The fungi were identified as Rhizopus stolonifer UAMH 114965 and Fusarium oxysporum UAMH 11966. These isolates have been deposited at the University of Alberta Microfungus Collection and Herbarium (UAMH), Toronto, CAN.

\section{Optimum temperature and water activity}

Rhizopus stolonifer grows at a faster rate than $F$. oxysporum (at each pairwise comparison at a particular temperature and water activity; $p<0.05$; Fig. 1). For $R$. stolonifer, the thermal growth range is $15-30^{\circ} \mathrm{C}$, and growth peaks at $20^{\circ} \mathrm{C}$. There is no growth at $35^{\circ} \mathrm{C}, 40^{\circ} \mathrm{C}$ and $45^{\circ} \mathrm{C}$. In contrast, $F$. oxysporum has broader thermal growth range $\left(15-35^{\circ} \mathrm{C}\right)$, higher optimum temperature $\left(25^{\circ} \mathrm{C}\right)$, and there is growth at $35^{\circ} \mathrm{C}$.

Both $R$. stolonifer and $F$. oxysporum can grow down to $0.95 \mathrm{a}_{\mathrm{w}}$, and they exhibit peak growth at $0.997 \mathrm{a}_{\mathrm{w}}$. There is no growth at $0.90,0.85,0.80,0.75,0.70,0.65,0.60,0.55,0.50$, and $0.40 \mathrm{a}_{\mathrm{w}}$ 's. Sporulation for $R$. stolonifer and $F$. oxysporum took place at any temperature and water activity where there was measurable growth. The exception was for $R$. stolonifer, where at $0.95 \mathrm{a}_{\mathrm{w}}$ and $15^{\circ} \mathrm{C} R$. stolonifer grew without producing aerial hyphae and subsequently no spores. At $15^{\circ} \mathrm{C}$, the minimum water activity for producing spores by $R$. stolonifer is $0.96 \mathrm{a}_{\mathrm{w}}$, and $0.95 \mathrm{a}_{\mathrm{w}}$ at higher temperatures like in F. oxysporum.

\section{Primary resource capture}

Radial growth rate of $R$. stolonifer was not reduced prior to hyphal contact by the addition of $F$. oxysporum within the same Petri dish (Table 1). Similarly, no decrease in radial growth rate by $F$. oxysporum occurred by the presence of $R$. stolonifer. No decrease in growth rate was observed in conspecific controls, as the result of when $R$. stolonifer and $R$. stolonifer, or $F$. oxysporum and $F$. oxysporum, were pitted against each other.

\section{Secondary resource capture}

Radial growth rate of $R$. stolonifer was reduced by $43 \%$ when it was placed on an existing, established mycelium of $F$. oxysporum (Table 2 ). However, $F$. oxysporum when added to the mycelium surface of an established $R$. stolonifer colony failed to initiate growth. Adding the same fungus to an existing mycelium of the same strain (conspecific controls) resulted in no measurable growth of the fungus that was introduced.

\section{Differential competition}

Rhizopus stolonifer spread over a greater surface area than $F$. oxysporum when they were placed in mixed proportions in a Petri dish (in each pairwise comparison, $p<0.05$; Fig. 2 ). The relative crowding coefficient (RCC) for the $R$. stolonifer $F$. oxysporum pair is 3.04. Increasing area of mycelium spread did not occur with increasing inoculum in a linear dose response: $y=125 x, R=$ 0.39 for $R$. stolonifer and $y=47 x, R=0.52$ for $F$. oxysporum. 


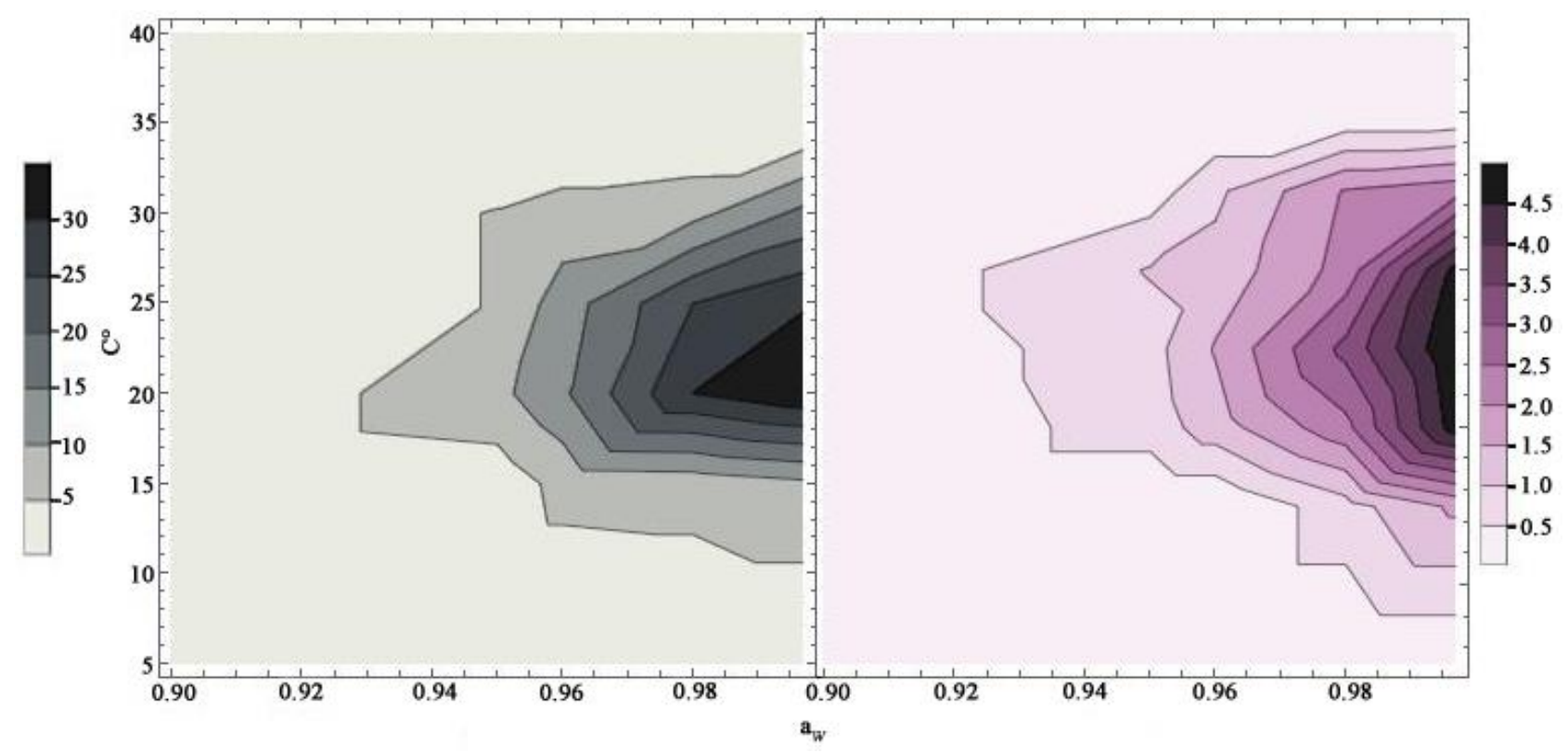

Fig. 1 - Contour plot of response surface showing optimization effects of temperature and water activity $\left(\mathrm{a}_{\mathrm{w}}\right)$ on growth of Rhizopus stolonifer UAMH 11965 (gray plot) and Fusarium oxysporum UAMH 11966 (purple plot). Radial growth rates are the mean $\mathrm{K}_{\mathrm{r}} \pm \mathrm{SE} \leq 0.34$.

\section{Discussion}

It is clear that $R$. stolonifer UAMH 11965 is the more aggressive, faster growing fungus (primary colonizer). As such, R. stolonifer UAMH 11965 is also an effective secondary colonizer, and $F$. oxysporum UAMH 11966 is the weaker, slower growing fungus of the pair; i.e., the RCC (relative crowding coefficient) is 3.0. The disproportionate growth favoring $R$. stolonifer results in large area of mycelium coverage at low inoculums (result of pairing a fast with a slow grower in the same dish); the response would otherwise be linear if growth rates of these fungi were similar, but this was not observed. Few, if any studies, report results for the direct fungus-fungus interaction in vitro for Rhizopus and Fusarium. We found that neither strain inhibited incoming hyphae when they were pitted against each other in the same Petri dish.

An established mycelium of $F$. oxysporum allowed for $R$. stolonifer to initiate growth, develop, establish, and produce spores, although at $43 \%$ the regular rate. At no time was the $F$. oxysporum replaced by $R$. stolonifer. Simply, $R$. stolonifer grew directly over $F$. oxysporum. Once past the edge of the $F$. oxysporum mycelium, where there is no inhibitory effect, the growth of $R$. stolonifer returned to its regular, fast rate (data not shown). Applying Lockwood's (1992) and Wicklow's (1992) interpretations, we conclude that $F$. oxysporum UAMH 11966 competes by interfering with $R$. stolonifer UAMH 11965 growth in order to secure limiting resources, whereas $R$. stolonifer UAMH 11965 is an exploitative competitor in addition to an interference competitor.

Contact with a wet (not damp) surface substrate is required for growth and sporulation by both $R$. stolonifer UAMH 11965 and $F$. oxysporum UAMH 11966. The minimum water activity $\geq 0.95 \mathrm{a}_{\mathrm{w}}$ for both of these shower-associated strains which is above the "wet", $0.90 \mathrm{a}_{\mathrm{w}}$ threshold as defined by Christian (1980). The growth of $R$. stolonifer and $F$. oxysporum peaks on agar that is close to water saturation. The optimum water activity for both species $=0.997 \mathrm{a}_{\mathrm{w}}$ and water $=1.00 \mathrm{a}_{\mathrm{w}}$. We found that "damp" conditions of 0.65-0.85 a will stop growth of $R$. stolonifer and F. oxysporum. Indeed, both Rhizopus and Fusarium are known for their habitat preference and suitability for moisture-rich substrates and surfaces (Amari et al. 2011). We now extend this trend to include Rhizopus and Fusarium isolates from a wet, shower substrate. A water-saturated wood substrate makes $F$. oxysporum and $R$. stolonifer successful, especially in a regularly used shower that would keep the moisture supply relatively continuous on a frequent basis. The growth of $R$. stolonifer UAMH 11965 and $F$. oxysporum UAMH 11966 is likely influenced by frequency and duration of showering that 
would contribute to the wood substrate holding more water for longer periods of time and less prone to evaporation and "dampness" which is unfavorable for fungus growth.

For the strains investigated here, F. oxysporum UAMH 11966 is high temperature adapted by having a higher upper lethal temperature of $35-40^{\circ} \mathrm{C}$ and higher optimum temperature of $25^{\circ} \mathrm{C}$ when compared to $R$. stolonifer UAMH 11965 . Thus, $F$. oxysporum can initiate growth, sporulate, and establish over a broader temperature range than $R$. stolonifer. Under $22-24^{\circ} \mathrm{C}$, indoor, standard comfort conditions for people (Ferng \& Lee 2002), the growth and sporulation of these showerassociated strains of $R$. stolonifer and $F$. oxysporum occur readily as long as surface substrates are "wet" to satisfy their water requirement. The mean $41^{\circ} \mathrm{C}$ preferred water temperature of a typical shower (Buijze et al. 2016) would be too high for $R$. stolonifer and $F$. oxysporum growth; however, adequate growth conditions would resume once the shower has ended. This strain of $F$. oxysporum UAMH 11966 has a competitive advantage over R. stolonifer UAMH 11965 under hotter conditions, at the upper end of temperature range for a typical mesophile (Amari et al. 2011).

Taken together, $R$. stolonifer and $F$. oxysporum (FOSC) are an antagonistic mycoflora, which consists of numerous non-pathogenic and pathogenic strains that can cause severe allergies and secondary infections in people (O'Donnell et al. 2009, Levetin et al. 2016, Egbuta et al. 2017). The importance of this study is that it examines, for the first time, the interaction in vitro between these two medically important molds that are specifically bathroom-associated isolates. This study identifies differences in mycelium growth, upper lethal temperature, and secondary resource capturing as the key survival elements that promote the natural coexistence and increase abundance of $F$. oxysporum (FOSC) UAMH 11966 and $R$. stolonifer UAMH 11965 in a shower setting.

Table 1 Primary resource capture experiments showing the radial growth of Rhizopus stolonifer UAMH 11965 and Fusarium oxysporum UAMH 11966 in paired competition experiments, 25 ${ }^{\circ} \mathrm{C}$. The mean radial growth rate $\left(\mathrm{K}_{\mathrm{r}}\right.$, the mean $\left.\pm \mathrm{SE}\right)$ was determined before hyphae of the two fungi made contact. Data followed by the same letter with a column are not significantly different $(p>$ $0.05)$.

\begin{tabular}{|c|c|c|c|}
\hline Competitor & Vs. & Test Fungus & $\mathrm{K}_{\mathrm{r}}(\mathrm{mm} / \mathrm{d})$ of Test Fungus \\
\hline None & & R. stolonifer & $28.7 \pm 0.3^{\mathrm{a}}$ \\
\hline R. stolonifer & & $R$. stolonifer & $31.4 \pm 0.4^{\mathrm{a}}$ \\
\hline F. oxysporum & & R. stolonifer & $31.2 \pm 0.4^{\mathrm{a}}$ \\
\hline None & & F. oxysporum & $5.2 \pm 0.3^{b}$ \\
\hline F. oxysporum & & F. oxysporum & $4.7 \pm 0.1^{b}$ \\
\hline R. stolonifer & & F. oxysporum & $5.3 \pm 0.2^{b}$ \\
\hline
\end{tabular}

Table 2 Secondary resource capture experiments showing the radial growth of Rhizopus stolonifer UAMH 11965 and Fusarium oxysporum UAMH 11966 when placed onto an existing mycelium of another fungus, $25^{\circ} \mathrm{C}$. NG, no growth. Data (the mean $\mathrm{K}_{\mathrm{r}} \pm \mathrm{SE}$ ) followed by the same letter with a column do not differ significantly $(p>0.05)$.

\begin{tabular}{lcc}
\hline \multicolumn{1}{c}{ Existing Mycelium } & Fungus Introduced & $\mathbf{K}_{\mathbf{r}}(\mathbf{m m} / \mathbf{d})$ of Fungus Introduced \\
\hline None & R. stolonifer & $29.2 \pm 0.5^{\mathrm{a}}$ \\
F. oxysporum & R. stolonifer & $16.6 \pm 0.4^{\mathrm{b}}$ \\
R. stolonifer & R. stolonifer & $\mathrm{NG}\left(0.0 \mathrm{~mm} / \mathrm{d}^{\mathrm{c}}\right)$ \\
& & \\
None & F. oxysporum & $4.9 \pm 0.3^{\mathrm{d}}$ \\
R. stolonifer & F. oxysporum & $\mathrm{NG}\left(0.0 \mathrm{~mm} / \mathrm{d}^{\mathrm{c}}\right)$ \\
F. oxysporum & F. oxysporum & $\mathrm{NG}\left(0.0 \mathrm{~mm} / \mathrm{d}^{\mathrm{c}}\right)$ \\
\hline
\end{tabular}




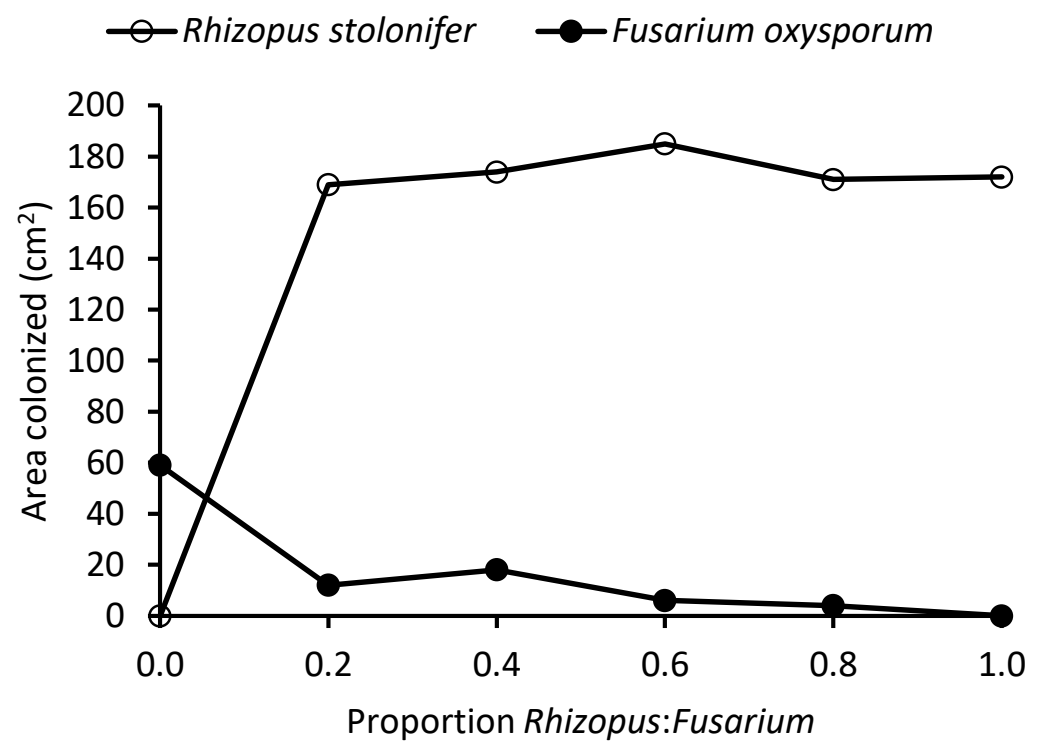

Fig. 2 - Differential competition colonizing ability Rhizopus stolonifer UAMH 11965 and Fusarium oxysporum UAMH $11966,25^{\circ} \mathrm{C}$, in mixed proportions in the same Petri dish. Each point is the mean $\pm \mathrm{SE} \leq 3.7$. The relative crowding coefficient (RCC) for this $R$. stolonifer-F. oxysporum pairing is 3.04.

\section{Acknowledgements}

Funded by a gift from Elizabeth E. Powelson to the Department of Biology for support of undergraduate research at Wittenberg University.

\section{References}

Amari A, Chai W, Schnabel G. 2011 - Effect of nutrient status, pH, temperature and water potential on germination and growth of Rhizopus stolonifer and Gilbertella persicaria. Journal of Plant Pathology 93, 603-612.

Baldrian P, Gabriel J. 2002 - Intraspecific variability in growth response to cadmium of the wood rotting fungus Piptoporus betulinus. Mycologia 94, 428-436.

Buijze GA, Sierevelt IN, van der Heijden BCJM, Dijkgraaf MG, Frings-Dresen MHW. 2016 - The effect of cold showering on health and work: A randomized controlled trial. PLoS- One. 11:e0161749.

Christian JHB. 1980 - Reduced water activity. In: Silliker JH, Elliott RP, Baird-Parker AC, Bryan FL, Christian JHB, Clark DS, Olson JC Jr, Roberts TA (eds), Microbial Ecology of Foods. Academic Press, New York, pp. 70-91.

de Almeida Júnior J, Ibrahim KY, Del Negro GM, Bezerra ED et al. 2016 - Rhizopus arrhizus and Fusarium solani concomitant infection in an immunocompromised host. Mycopathologia 181, $125-129$.

Egbuta MA, Mwanza M, Babalola OO. 2017 - Health risks associated with exposure to filamentous fungi. International Journal of Environmental Research and Public Health 14, 719-735.

Ferng SF, Lee LW. 2002 - Indoor air quality assessment of daycare facilities with carbon dioxide, temperature, and humidity as indicators. Journal of Environmental Health 65, 14-18.

Garner D, Machin K. 2008 - Investigation and management of an outbreak of mucormycosis in a paediatric oncology unit. Journal of Hospital Infection 70, 53-59.

Hamada N, Abe N. 2009 - Physiological characteristics of 13 common fungal species in bathrooms. Mycoscience 50, 421-429. 
Hamada N, Abe N. 2010a - Comparison of fungi found in bathrooms and sinks. Biocontrol Science $15,51-56$.

Hamada N, Abe N. 2010b - Growth characteristics of four fungal species in bathrooms. Biocontrol Science 15, 111-115.

Hernádez-Lauzardo A, Bautista-Baños S, Velázquez del Valle M, Trejo-Espino J. 2005 Identification of Rhizopus stolonifer (Ehrenb.: Fr.) vuill., causal agent of Rhizopus rot disease of fruits and vegetables. Revista mexicana de fitopatología 24, 65-69.

Ibrahim M, Shehu K, Tafinta IY, Iman UA, Hassana YI. 2014 - Efficacy of some plant extracts on growth and germination of Rhizopus stolonifer and Fusarium oxysporum isolated from rotten Irish potato tubers. Annals of Biological Science 2, s63-67.

Levetin E, Horner WE, Scott JA. 2016 - Taxonomy of allergenic fungi. Journal of Allergy and Clinical Immunology in Practice 4, 375-385.

Lockwood JL. 1992 - Exploitation competition. In: Carroll GC, Wicklow DT (eds), The Fungal Community - Its Organization and Role in the Ecosystem, 2nd ed. Marcel Dekker, New York, pp. 243-263.

O’Donnell K, Gueidan C, Sink S, Johnston PR et al. 2009 - A two-locus DNA sequence database for typing plant and human pathogens within the Fusarium oxysporum species complex. Fungal Genetics and Biology 46, 936-948.

Rafique K, Rauf CA, Naz F, Shabbir G. 2015 - DNA sequence analysis, morphology and pathogenicity of Fusarium oxysporum f. sp. lentis isolates inciting lentil wilt in Pakistan. International Journal of Biosciences 7, 74-91.

Rousseau S, Donèche B. 2001 - Effect of water activity $\left(a_{w}\right)$ on the growth of some epiphytic microorganisms isolated from grape berry. Vitis 40, 75-78.

Short DP, O'Donnell K, Zhang N, Juba JH, Geiser DM. 2011 - Widespread occurrence of diverse human pathogenic types of the fungus Fusarium detected in plumbing drains. Journal of Clinical Microbiology 49, 4246-4272.

Wicklow DT. 1992 - Interference competition. In: Carroll GC, Wicklow DT (eds), The Fungal Community - Its Organization and Role in the Ecosystem, 2nd ed. Marcel Dekker, New York, pp. 265-274. 